\title{
Stepwise Arms Race Between AvrPik and Pik Alleles in the Rice Blast Pathosystem
}

\author{
Weihuai Wu, ${ }^{1,2}$ Ling Wang, ${ }^{1}$ Shu Zhang, ${ }^{1}$ Zekang Li, ${ }^{1}$ Yu Zhang, ${ }^{1}$ Fei Lin, ${ }^{1}$ and Qinghua Pan ${ }^{1}$ \\ ${ }^{1}$ National Key Laboratory for Conservation and Utilization of Subtropical Agrobioresurces and Guangdong Provincial Key \\ Laboratory for Microbe Signals and Crop Disease Control, South China Agricultural University, Guangzhou, 510642, China; \\ ${ }^{2}$ Hainan Key Laboratory for Monitoring and Control of Tropical Agricultural Pests, Environment and Plant Protection Institute, \\ Chinese Academy of Tropical Agricultural Sciences, Haikou, Hainan 571101, China
}

Submitted 17 February 2014. Accepted 1 April 2014.

\begin{abstract}
A stepwise mutation that occurred in both pathogens and their respective hosts has played a seminal role in the coevolutionary arms race evolution in diverse pathosystems. The process driven by rice blast AvrPik and Pik alleles was investigated through population genetic and evolutionary approaches. The genetic diversity of the non-signal domain of AvrPik was higher than that in its signal peptide domain. Positive selection for particular AvrPik alleles in the northeastern region of China was stronger than in the south. The perfect relationship between the functional lineages and AvrPik allele-specific pathotypes was established by ruling out the nonfunctional lineages derived from additional copies. Only four alleles conditioning stepwise pathotypes were detected in natural populations, which were likely created by only one evolutionary pathway with three recognizable mutation steps. Two non-stepwise pathotypes were determined by two blocks in a network constructed by all 16 possible alleles, indicating that a natural evolution process can be artificially changed by a combination of specific single-nucleotide polymorphisms. Assuming that AvrPik evolution has been largely driven by host selection, the coevolutionary stepwise relationships between AvrPik and Pik was established. The experimental validation of stepwise mutation is required for the development of sustainable management strategies against plant disease.
\end{abstract}

The co-evolution between a pathogen and its host involves the continuous development by the host of novel resistance $(R)$ genes, which prompts attempts by the pathogen to overcome them. The process is discernible at the level of the genome in both the host and the pathogen (Kizinger and Stavrinides 2012; Raffaele et al. 2010; Terauchi and Yoshida 2010). Novel alleles can arise in the pathogen via mutation or gene flow; however, this aspect of the pathogen genome is strongly affected by host selection (Daugherty and Malik 2012; Kanzaki et al. 2012; Stukenbrock and McDonald 2009). Identifying the genetic structures of pathogen populations has relied in the past mainly on characterizing the pathotypes present in a popula-

$\mathrm{W}$. Wu, L. Wang, and S. Zhang contributed equally to this work.

Corresponding author: Q. Pan; Telephone: +86-20-85288315; E-mail: panqh@scau.edu.cn

* The $\boldsymbol{e}$-Xtra logo stands for "electronic extra" and indicates that three supplementary figures and four supplementary tables are published online.

(C) 2014 The American Phytopathological Society tion, as inferred from the response of a differential set of host cultivars known to differ with respect to the profile of $R$ genes that they harbor (Hamer 1991). The procedure is somewhat cumbersome and time consuming but has now become replaceable, at least in part, by DNA fingerprinting (Hamer 1991; McDonald 1997; Schürch et al. 2004).

The type of molecular marker preferred for genotyping has shifted from dispersed repetitive DNA sequences (Hamer 1991; Jiménez-Gasco et al. 2004) to various polymerase chain reaction (PCR) assays (Mahuku et al. 2002; Steele et al. 2001). A weakness of all of these fingerprinting technologies is that they do not target variation in specific genes and, thus, are incapable of reflecting the pathogenicity profile of the disease organism (Mahuku et al. 2002). Within-gene assays can, in principle, be developed based on either insertion-deletion or single-nucleotide variation (Champouret et al. 2009; Dai et al. 2010; Schürch et al. 2004; Tosa et al. 2005). However, little progress has been made to date in establishing robust relationships between genotype and pathotype, despite the wealth of research effort already applied to pathogen DNA fingerprinting (Champouret et al. 2009; Dai et al. 2010; Jiménez-Gasco et al. 2004; Tosa et al. 2005; Zeigler et al. 1995).

Pathogens capable of overcoming multiple $R$ genes are thought to evolve by accumulating the appropriate mutations in a stepwise fashion in many pathosystems (Hovmøller and Justetson 2007; Jiménez-Gasco et al. 2004; Kiyosawa 1982; Lozovsky et al. 2009; Sirawaraporn et al. 1997; Steele et al. 2001). An example of this process has been provided by the behavior of the wheat fungal pathogen Puccinia striiformis $\mathrm{f}$. sp. tritici, in which changes from avirulence to virulence were associated with the gain or loss of a specific amplified fragment length polymorphism fragment (Hovmøller and Justetson 2007). Analysis of malaria parasite (Plasmodium falciparum) mutants has suggested that multiple mutants can emerge from a single mutant via stepwise selection (Sirawaraporn et al. 1997), based on a small number of possible pathways (Lozovsky et al. 2009). Whether an analogous situation characterizes host selection during a co-evolutionary arms race is not known.

Rice blast, a potentially devastating disease of rice, is caused by the filamentous ascomycete fungus Magnaporthe oryzae. A series of alleles at the major rice blast $R$ gene locus $P i k$, which were introduced from Chinese (Pik, Pik-m, and Pik-s), Indian (Pik-h), and Pakistani (Pik-p) landraces in the 1960s (Kiyosawa 1974), are known to condition a stepwise and broader resistance spectra, and these have been heavily exploited as sources of genetic resistance in a number of rice breeding programs since the 1970s (Hua et al. 2012; Kiyosawa 1982; Wang et al. 2009; Xing et al. 1991; Zhai et al. 2011). On the other hand, 
stepwise mutations of AvrPik alleles were also observed in the early 1970s (Kiyosawa 1982). Recently, Yoshida and his colleagues have successfully isolated the AvrPik via association genetics approach, and then found that there are five alleles related to the combinations of the five amino acids (AvrPik-A to AvrPik-E) (Yoshida et al. 2009). Furthermore, they have attempted to prove the arms race co-evolution relationships between AvrPik and Pik alleles by a protein-protein interaction method (Kanzaki et al. 2012). The rice-M. oryzae interaction is a typical gene-for-gene pathosystem; therefore, the various host resistance specificities expressed by the fungal avirulence (Avr) gene AvrPik are likely to have evolved from a common progenitor in a stepwise fashion. Our objective in this research was to investigate the genetic structures of AvrPik alleles in various pathogen populations, and to use these data to reconstruct the evolution of the gene in terms of the identity of the $P i k$ alleles, which it likely encountered.

\section{RESULTS}

\section{AvrPik pathotypes.}

Four stepwise pathotypes (namely AAAA, VAAA, VVAA, and VVVV), in which the first to fourth infection types corresponded to Pik-p, Pik, Pik-m, and Pik-h, respectively, were identified among the 240 blast isolates (four populations) (Supplementary Table S1; Fig. 1A). The frequency of the AAAA pathotype was highest in the southern part of China (66.7\% in Guangdong [GD] province and $61.7 \%$ in Hunan $[\mathrm{HN}])$, was moderate $(46.5 \%)$ in the northeastern province of Liaoning (LN), and was just $8.8 \%$ in the far northeast (Heilongjiang [HLJ] province); the frequency of the most virulent pathotype, VVVV, increased in the opposite direction $3.3 \%$ in $\mathrm{GD}, 6.6 \%$ in $\mathrm{HN}, 41.4 \%$ in $\mathrm{LN}$, and $82.5 \%$ in HLJ). Correspondingly, the resistance spectra of the four Pik alleles were stepwise as $P i k-p<P i k<P i k-m$ or $P i k-h$ in all four populations (Fig. 1B). Interestingly, the integral level of resistance of the four Pik alleles was further decreasingly stepwise from the southern to northeastern regions (Fig. 1B). Taken together, the complementary and stepwise frequencies of both virulence (AAAA and VVVV) and resistance (all four Pik alleles) indicated that the host selection on the AvrPik alleles was steadily increased from southern to northeastern regions.

\section{AvrPik genotypes.}

Four primer sets $(136 \mathrm{~F} / \mathrm{R}, 139 \mathrm{~F} / \mathrm{R}, 143 \mathrm{~F} / \mathrm{R}$, and 234F/R) were designed to evaluate the four main single-nucleotide polymorphisms (SNP) - C136A, C139G, G143A, and G234A, respectively (Yoshida et al. 2009)—via derived cleaved amplified polymorphic sequence (dCAPS) analysis (Fig. 2). In all, 14 distinct AvrPik genotypes were identified in the four populations; three of those $(1113,2123$, and 3113$)$ were widely distributed geographically, while the other 11 were region-specific (Table 1; Fig. 2). The isolates split roughly 1:1 in terms of those carrying common (114 isolates) and those carrying region-specific (126 isolates) AvrPik genotypes. Among the latter, approximately half were from the southern region (58 isolates) and half from the northeastern region (62 isolates) (the remaining six isolates were detected in both regions) (Table 1). Furthermore, there were only four genotypes in GD, six in $\mathrm{HN}, 10$ in JL, and 11 in HLJ, indicating that AvrPik alleles were more diverse in the northeastern than in the southern regions.

\section{Genetic diversity of the AvrPik alleles.}

The genetic diversity at the AvrPik locus among the four populations was explored in some detail by resequencing a subset of 60 isolates. The AvrPik coding region harbored 18 SNP, of which seven were synonymous (data not shown). In all, four of the 11 above-mentioned nonsynonymous SNP were ubiquitous and have been noted previously (Yoshida et al. 2009). These are referred to hereafter as "major" SNP, and the remainder as "minor" ones. The non-signal domain harbored 16 of the SNP sites, while the other two were within the signal

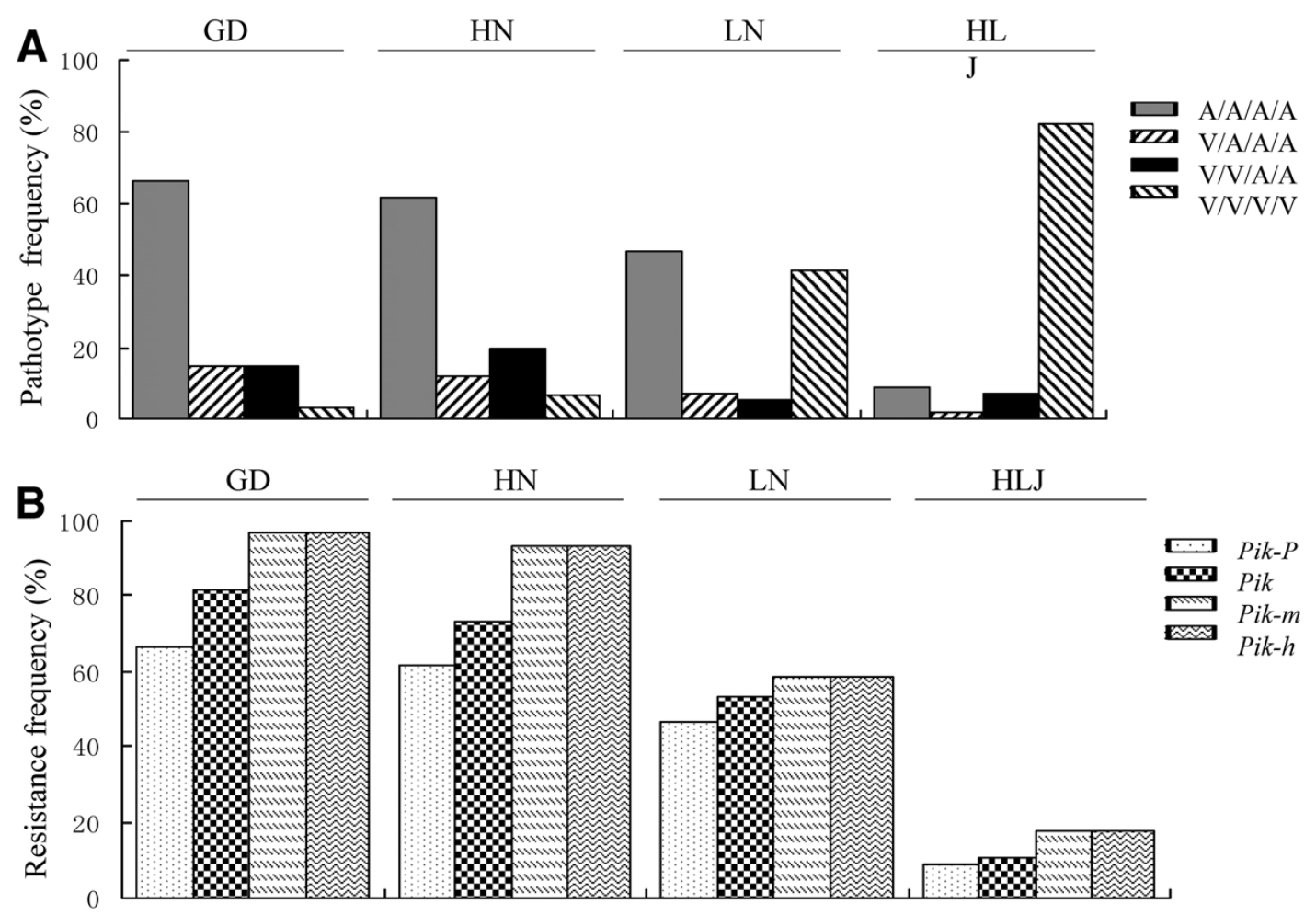

Fig. 1. A, Pathotype and B, resistance frequencies of AvrPik and Pik alleles, respectively, in Magnaporthe oryzae populations collected from Guangdong (GD), Hunan (HN), Liaoning (LN), and Heilongjiang (HLJ) provinces, China. Sixty isolates each from GD, HN, LN, and HLJ were subjected to pathotyping based on four Pik monogenic lines: IRBLkp-K60 (Pik-p), IRBLk-Ka (Pik), IRBLkm-TS (Pik-m), and IRBLkh-K3 (Pik-h). 
peptide domain; as a result, the level of nucleotide diversity in the former domain (0.007) was higher than in the latter $(0.001)$ (Table 2). In total, three of the variable sites were found in the GD population, four in the HN population, and 10 each in the LN and HLJ populations. The levels of nucleotide diversity at the population level were $0.002,0.003,0.008$, and 0.008 , respectively. Therefore, the AvrPik sequence appeared to be more diverse in the northeastern than in the southern populations. As a control, only one polymorphic nucleotide of the neutral gene, MoDUO1 (Peng et al. 2011), was found in isolate CHL2394 within the subset (data not shown).

\section{Selection at the AvrPik locus.}

The Fu and $\mathrm{Li}$ (1993) tests for selection and neutrality were performed to assess the evolutionary processes acting on AvrPik. Both $D^{*}$ and $F^{*}$ were negative and significantly different from neutrality in both the signal and non-signal domains, and the values suggested that a stronger selection pressure was being imposed on the non-signal peptide domain (Table 2). Both parameters were positive in the $\mathrm{HN}$ population but negative in the other three populations; however, they did not deviate significantly from neutrality in any of the four populations (Table
2). The nonsynonymous/synonymous $(\mathrm{Ka} / \mathrm{Ks})$ ratio was 5.2 across the entire coding region and 4.7 in the non-signal peptide region (Table 2), consistent with a stronger positive selection pressure having been imposed on the coding region. $\mathrm{Ka} / \mathrm{Ks}$ was 1.7 for the GD and 2.3 for the $\mathrm{HN}$ population but 7.5 and 6.5 for the LN and HLJ populations, respectively, again implying that there has been a stronger selection pressure on AvrPik in the northeast than in the south of China.

\section{Phylogeny of the AvrPik alleles.}

A phylogeny was constructed based on the 78 coding sequences present in 60 isolates, of which 18 were of genotypes $2113,2123,2223$, and 2232 that have two sequences with the respective point mutations. Four lineages (I to IV) were recognizable, correlating with the four major SNP and resulting in the four major variations $(\mathrm{H} 46 \mathrm{~N}, \mathrm{P} 47 \mathrm{~A}, \mathrm{G} 48 \mathrm{D}$, and M78I) at the amino acid level (Table 3). That is, the four lineages were correlated to the four major genotypes (alleles) of AvrPik. On the basis of the other SNP, the four lineages could be subdivided into Ia-d, IIa and IIb, IIIa, and IVa-d (Table 3). The predominant sublineages were Ia, IIa, IIIa, and IVa (Table 3).

A
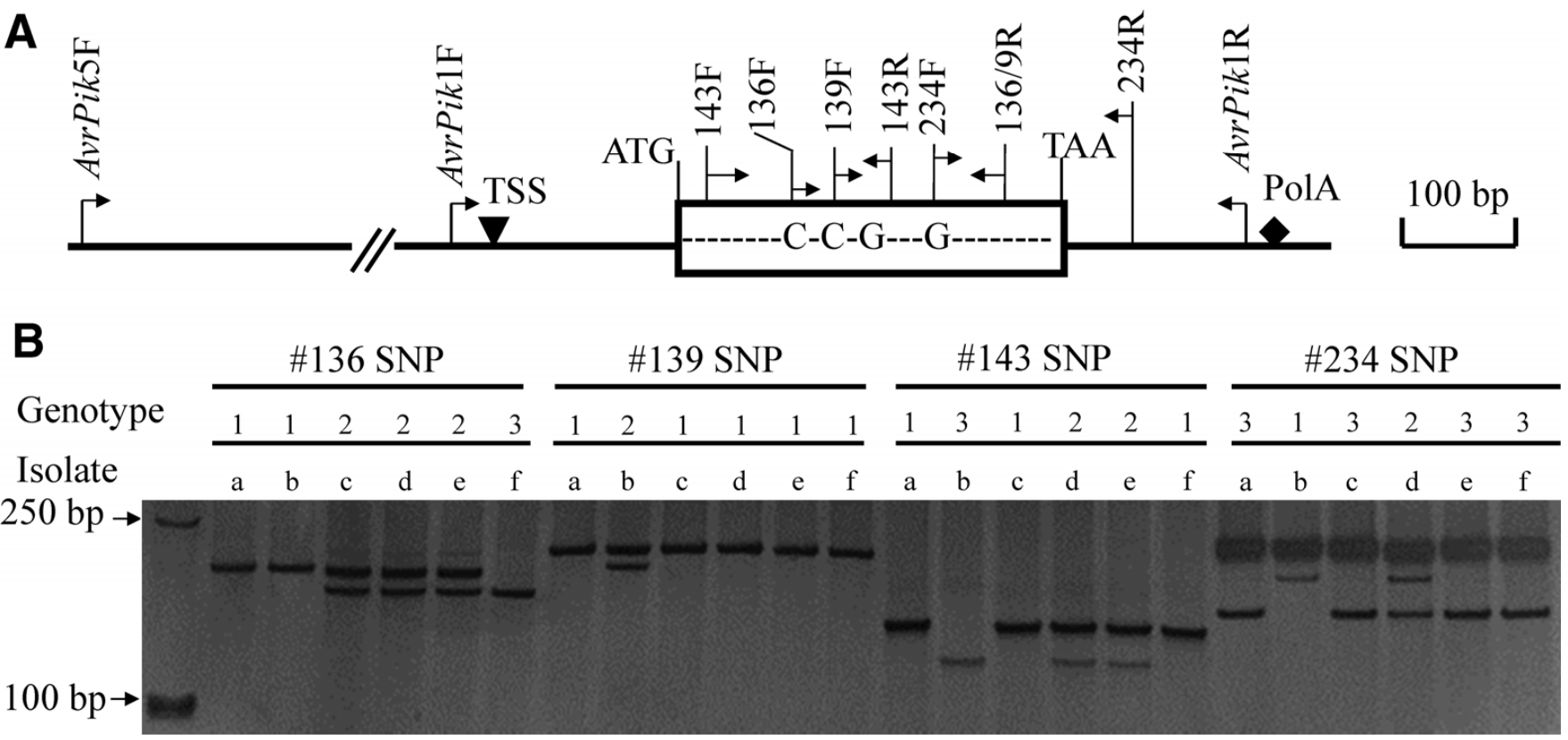

Fig. 2. Genotyping of isolates of Magnaporthe oryzae via integration of the four major single-nucleotide polymorphisms (SNP) of the AvrPik alleles. A, Schema for genotyping of the AvrPik alleles. The primer set AvrPik1F/1R was used to detect the presence or absence of the AvrPik alleles. Four primer sets (136F/R, 139F/R, 143F/R, and 234F/R) were designed to evaluate number $136 \mathrm{SNP}$ (C/A), number $139 \mathrm{SNP}$ (C/G), number 143 SNP (G/A), and number 234 SNP (G/A), respectively, via derived cleaved amplified polymorphic sequence analysis. The primer set AvrPik5F/143R was used to detect traansposable element in the $5^{\prime}$ region. The coding region is represented by open box, and the initiator and terminator were also indicated. B, Six isolates (a to f) belonging to the six genotypes were selected for the four rounds of SNP assay, thereby determining the genotype of each isolate by four-digit code: a, 1113 (EHL0338); b, 1231 (EHL0519); c, 2113 (EHL0979); d, 2122 (EHL0919); e, 2123 (CHL2384); and f, 3113 (CHL2446). Numerical codes: 1, polymerase chain reaction (PCR) product that cannot be digested by the respective enzyme, 2, PCR product that can be partially digested, and 3, PCR product that can be completely digested.

Table 1. Distribution of the integrated genotypes of AvrPik of Magnaporthe oryzae in the rice blast populations collected from Guangdong (GD), Hunan (HN), Liaoning (LN), and Heilongjiang (HLJ) provinces, China

\begin{tabular}{|c|c|c|c|c|c|c|c|c|c|c|c|c|c|c|c|}
\hline \multirow[b]{2}{*}{ Population } & \multicolumn{14}{|c|}{ Integrated genotype $^{a}$} & \multirow[b]{2}{*}{ Total } \\
\hline & 1113 & 1121 & 1123 & 1231 & 1233 & 2113 & 2121 & 2122 & 2123 & 2223 & 2231 & 2232 & 2233 & 3113 & \\
\hline GD & 18 & $\ldots$ & $\ldots$ & $\ldots$ & $\ldots$ & 35 & $\ldots$ & $\ldots$ & 4 & $\ldots$ & $\ldots$ & $\ldots$ & $\ldots$ & 3 & 60 \\
\hline $\mathrm{HN}$ & 19 & $\ldots$ & 1 & $\ldots$ & $\ldots$ & 23 & $\ldots$ & $\ldots$ & 11 & 2 & $\ldots$ & $\ldots$ & $\ldots$ & 4 & 60 \\
\hline $\mathrm{LN}$ & 2 & $\ldots$ & $\ldots$ & 14 & 1 & $\ldots$ & 1 & 1 & 34 & 1 & $\ldots$ & 1 & 1 & 4 & 60 \\
\hline HLJ & 5 & 2 & 1 & 30 & 1 & $\ldots$ & 3 & 3 & 9 & 1 & 4 & $\ldots$ & $\ldots$ & 1 & 60 \\
\hline Total & 44 & 2 & 2 & 44 & 2 & 58 & 4 & 4 & 58 & 4 & 4 & 1 & 1 & 12 & 240 \\
\hline Isolates $(\%)$ & 18.3 & 0.8 & 0.8 & 18.3 & 0.8 & 24.2 & 1.7 & 1.7 & 24.2 & 1.7 & 1.7 & 0.4 & 0.4 & 5.0 & $\ldots$ \\
\hline
\end{tabular}

${ }^{a}$ Four-digit code describing genotype: the first code represents number 136 single-nucleotide polymorphism (SNP), the second number 139 SNP, the third number 143 SNP, and the fourth number 234 SNP. 


\section{Relationships between the functional lineages and the four stepwise pathotypes.}

The poor correlations between lineages and the AvrPik allele-specific pathotypes were observed when all 78 alleles of AvrPik were included but the perfect relationships were constructed when only 60 alleles were used (Table 3), indicating that there were functional (dominant or epistatic) and nonfunctional (recessive or hypostatic) lineages (alleles), with the 18 alleles of the latter one being ruled out (Supplementary Fig. S1, 'Lineage', 'Functional lineage', and 'Pathotype'). That is, 18 of 60 isolates resequenced have a duplication of the locus. As a result, there were 11 functional lineages for the 60 isolates, which is completely associated with the respective pathotypes (Table 3 ). Among the four major sublineages, Ia (genotype HPGM) matched pathotype AAAA (carried by 24 isolates, all of which were avirulent against all four Pik alleles); IIa (genotype NPGM) matched pathotype VAAA (13 isolates, virulent against Pik- $p$ but avirulent against $P i k, P i k-m$, and $P i k-h)$; IIIa (genotype NADM) matched pathotype VVAA (four isolates virulent against $P i k-p$ and $P i k$ but avirulent against $P i k-m$ and $P i k-h$ ); and, finally, IVa (genotype NADI) matched pathotype VVVV (11 isolates, virulent against all four Pik alleles) (Table 3). As for the remaining seven minor sublineages, all of them matched the same pathotype VVVV based on various criteria, which were confirmed by resequencing and artificial mutant reconstruction (Table 3; Supplementary Tables S2 and S3). Both sublineages Ib and Ic (genotype HPGM) were due to transposable element (TE) insertion in their promoter regions but not to the minor mutations D90N and T69A, respectively, because both mutations did not affect the pathotypes; Id (genotype HPGM) was due to a mutation causing a premature stop codon at the number 56 position; IIb was due to the point mutation A67D with an additive effect on KM2 (genotype NPGM); and IVb, IVc, and IVd were due to additional point mutations (I33T, L11P, and A32S, respectively) that occurred on KM16 (genotype NADI), with their effects not recognizable by the four host monogenic lines (Table 3).

Table 2. Evolutionary parameters associated with the AvrPik alleles in four rice blast populations collected from Guangdong (GD), Hunan (HN), Liaoning (LN), and Heilongjiang (HLJ) provinces, China

\begin{tabular}{|c|c|c|c|c|c|c|c|}
\hline Domain, population & $S^{\mathbf{a}}$ & $\pi^{\mathbf{b}}$ & $D^{* \mathrm{c}}$ & $F^{* \mathrm{c}}$ & $K a^{\mathrm{d}}$ & $K s^{\mathrm{e}}$ & $K a / K s$ \\
\hline \multicolumn{8}{|l|}{ Domain } \\
\hline Entire coding region & 18 & 0.006 & $-4.21 * *$ & $-3.75 * *$ & 22.2 & 4.3 & 5.2 \\
\hline Non-signal-peptide region & 16 & 0.007 & $-3.79 * *$ & $-3.37 * *$ & 26.1 & 5.6 & 4.7 \\
\hline Signal peptide region & 2 & 0.001 & $-2.73^{*}$ & $-2.71^{*}$ & 3.4 & 0 & $K a>K s$ \\
\hline \multicolumn{8}{|l|}{ Population } \\
\hline GD & 3 & 0.002 & -1.31 & -1.21 & 0.5 & 0.3 & 1.7 \\
\hline $\mathrm{HN}$ & 4 & 0.003 & 0.16 & 0.02 & 0.7 & 0.3 & 2.3 \\
\hline $\mathrm{LN}$ & 10 & 0.008 & -1.34 & -1.22 & 1.5 & 0.2 & 7.5 \\
\hline HLJ & 10 & 0.008 & -0.84 & -0.79 & 1.3 & 0.2 & 6.5 \\
\hline
\end{tabular}

a Number of segregating sites.

b Nei's nucleotide diversity based on silent site.

${ }^{\mathrm{c}} \mathrm{Fu}$ and $\mathrm{Li}$ 's $D^{*}$ and $F^{*}$; ${ }^{*}$ and $* *$ represent statistical significance at $P<0.05$ and 0.01 levels, respectively.

d Nonsynonymous.

e Synonymous.

Table 3. Amino acid differences within AvrPik products generated by 60 rice blast isolates, and the relationships between functional lineages and pathotypes

\begin{tabular}{|c|c|c|c|c|c|c|c|c|c|c|c|c|c|c|}
\hline \multirow[b]{2}{*}{ Lineage } & \multirow[b]{2}{*}{ Isolate } & \multicolumn{11}{|c|}{ Mutated amino acids and their positions ${ }^{a}$} & \multirow[b]{2}{*}{ Pathotype $^{b}$} & \multirow[b]{2}{*}{ Mutant $^{\mathrm{c}}$} \\
\hline & & 11 & 32 & 33 & 46 & 47 & 48 & 56 & 67 & 69 & 78 & 90 & & \\
\hline & Ina168 (reference) & $\mathrm{L}$ & A & I & $\mathrm{H}$ & $\mathrm{P}$ & $\mathrm{G}$ & $\mathrm{W}$ & A & $\mathrm{T}$ & M & $\mathrm{D}$ & $\ldots$ & $\ldots$ \\
\hline Ia & $\begin{array}{l}\text { CHL2370, CHL2384, CHL2409, CHL2412, CHL2446, } \\
\text { CHL2492, CHL2560, CHL2561, CHL2608, CHL2636, } \\
\text { EHL0312, EHL0317, EHL0326, EHL0346, EHL0363, } \\
\text { EHL0377, EHL0379, EHL0489, EHL0496, EHL0524, } \\
\text { EHL0624, EHL0918, EHL0963, EHL0979 }\end{array}$ & - & - & - & • & • & - & - & - & · & - & - & $\mathbf{A} / \mathbf{A} / \mathbf{A} / \mathbf{A}$ & KM1 \\
\hline Ib & EHL0961 & . & . & . & $\cdot$ & . & . & . & . & A & . & $\cdot$ & $\mathrm{V} / \mathrm{V} / \mathrm{V} / \mathrm{V}$ & KM22 \\
\hline Ic & EHL0794 & . & . & . & . & . & . & . & . & . & . & $\mathrm{N}$ & $\mathrm{V} / \mathrm{V} / \mathrm{V} / \mathrm{V}$ & KM17 \\
\hline Id & EHL0931 & . & . & . & . & . & . & $*$ & & & & & $\mathrm{~V} / \mathrm{V} / \mathrm{V} / \mathrm{V}$ & N/A \\
\hline IIa & $\begin{array}{l}\text { CHL2345, CHL2394, CHL2593, CHL2606, CHL2631, } \\
\text { EHL0331, EHL0338, EHL0339, EHL0382, EHL0386, } \\
\text { EHL0880, EHL0882, EHL0988 }\end{array}$ & - & · & - & $\mathbf{N}$ & - & - & · & - & - & - & · & V/A/A/A & KM2 \\
\hline $\mathrm{IIb}$ & EHL0559, EHL0866 & . & . & . & $\mathbf{N}$ & . & . & . & $\mathrm{D}$ & . & . & . & $\mathrm{V} / \mathrm{V} / \mathrm{V} / \mathrm{V}$ & KM18 \\
\hline IIIa & EHL0471, EHL0486, EHL0621, EHL0821 & . & $\cdot$ & . & $\mathbf{N}$ & A & $\mathbf{D}$ & . & . & . & . & . & V/V/A/A & KM12 \\
\hline IVa & $\begin{array}{l}\text { EHL0396, EHL0447, EHL0472, EHL0519, EHL0555, } \\
\text { EHL0592, EHL 0615, EHL0750, EHL0858, EHL0917, } \\
\text { EHL0937 }\end{array}$ & . & . & . & $\mathbf{N}$ & $\mathbf{A}$ & D & . & . & . & $\mathbf{I}$ & $\cdot$ & $\mathbf{V} / \mathbf{V} / \mathbf{V} / \mathbf{V}$ & KM16 \\
\hline IVb & EHL0635 & · & $\cdot$ & $\mathrm{T}$ & $\mathbf{N}$ & $\mathbf{A}$ & D & . & . & . & I & . & $\mathrm{V} / \mathrm{V} / \mathrm{V} / \mathrm{V}$ & KM19 \\
\hline IVc & EHL0547 & $\mathrm{P}$ & . & . & $\mathbf{N}$ & $\mathbf{A}$ & D & . & . & . & I & . & $\mathrm{V} / \mathrm{V} / \mathrm{V} / \mathrm{V}$ & KM21 \\
\hline IVd & EHL0849 & · & $\mathrm{S}$ & . & $\mathbf{N}$ & $\mathbf{A}$ & D & . & . & . & I & $\cdot$ & $\mathrm{V} / \mathrm{V} / \mathrm{V} / \mathrm{V}$ & KM20 \\
\hline
\end{tabular}

${ }^{a}$ Residues shown in bold are the output of major single-nucleotide polymorphisms, dots indicate matching residues with the peptide sequence of isolate Ina168, and * indicates the premature stop codon present in isolate EHL0931.

b Pathotype with respect to Pik-p, Pik, Pik-m, and Pik-h. Those shown in bold indicate the major pathotype corresponding to the major lineage.

${ }^{\mathrm{c}}$ Mutation reconstructed. Four major (KM1, KM2, KM12, and KM16) and six minor (KM17 to KM22) mutants were artificially reconstructed. 
An evolutionary network of the global AvrPik alleles.

The four major SNP at AvrPik could theoretically give rise to 16 genotypic combinations. However, only four major genotypes were found in nature. To investigate the evolutionary pathways of the four major genotypes conditioning stepwise virulence, all 16 possible alleles (assigned KM1 to KM16 with allele codes from 0000 to 1111) were created by site-directed mutagenesis and, then, subjected to pathotype testing (Fig. 3A). A network of these 16 alleles comprising 24 pathways from 0000 to 1111 via four evolutionary phases (defined as one, two, three, and four SNP were mutated from 0 to 1 ) was constructed (Fig. 3A). There were 32 single SNP pairs in the network, of which 21 conditioned the different pathotypes (e.g., 0000/1000, 1000/1100, and 0001/0011), and 11 conditioned the same pathotypes (e.g., 0000/0100, $1100 / 1110$, and 0011/0111). Such SNP in the former and latter cases have made additive and cumulative contributions, respectively, to race specificity. As for the individual allele, including the artificial one, five produced an AAAA pathotype, one VAAA, three VVAA, and two VVVV. Interestingly, three and two artificial alleles in two blocks produced the nonstepwise pathotypes AVAA and VVAV, respectively, (Fig. 3A; Supplementary Fig. S2). None of the alleles produced pathotype VVVA, a type which was also not represented in any of the four populations. Unexpectedly, there was no allele on phase II in the four natural populations. Thus, just three mutation steps were required to evolve the four major AvrPik alleles as $\operatorname{HPGM}(0000)>\operatorname{NPGM}(1000)>\operatorname{NADM}(1110)>\mathrm{NADI}$

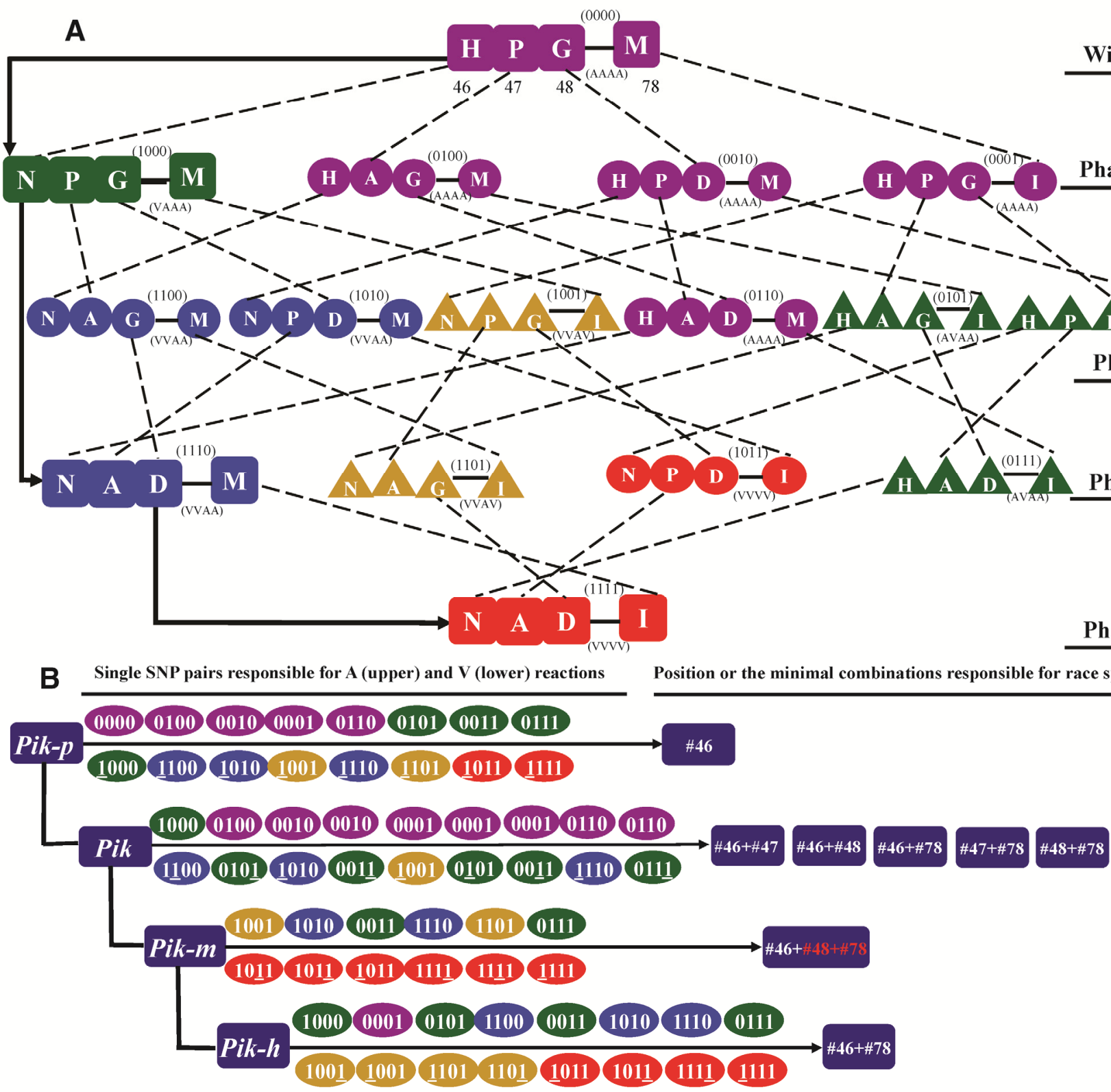

Fig. 3. Reconstruction of all 16 possible AvrPik alleles with combinations of the four major single-nucleotide polymorphisms (SNP). A, An evolutionary network of the 16 possible AvrPik alleles corresponding to the four host Pik alleles. The 16 AvrPik alleles in the network are as follows: squares, the natural alleles; circles, the artificial alleles with stepwise phenotypes; and triangles, the artificial alleles with non-stepwise phenotypes. The six pathotypes (corresponding to Pik-p, Pik, Pik-m, and Pik-h) detected in the network are as follows: purple, 4A (AAAA); green, 3A (VAAA and AVAA): blue, 2A (VVAA), yellow, 1A (VVAV); red, 0A (VVVV). The wild type (0000; AAAA) to the most virulent type (1111; VVVV) were processed by the four-phase-mutations. Three-stepwise-natural mutations (bold arrow) were also indicated. B, Determinants of AvrPik allele specificity. SNP (underlined) pairs determining specificity against each $P i k$ allele are assembled and the positions or the minimal combinations responsible for race specificity are presumed. 
(1111), which were likely on the one evolutionary pathway that omitted Phase II in nature.

\section{The determinants of AvrPik allele specificities.}

To find out which positions determine AvrPik allele specificity, single SNP pairs, which differentiate between virulent and avirulent infection types against the specific Pik alleles, were assembled (Fig. 3B). Eight pairs were involved for Pik-p but, because only the number 46 position varied within pairs, this is the definitive determinant of specificity against this $R$ gene. The nine pairs associated with virulence for Pik involved all four major SNP, so that one of the five minimal combinations of two SNP (numbers $46+47,46+48,46+78,47+78$, and $48+78$ ) may be the determinant for Pik specificity. All six pairs associated with Pik-m involved numbers 46, 48, and 78, suggesting that the minimal combination $46+48+78$ is the most likely determinant for Pik-m specificity. Finally, because positions 46 and 78 were involved in all eight $P i k$ - $h$ pairs, the minimal combination $46+78$ was thought to be the most likely determinant.

\section{Interactions between AvrPik and Pik allelic proteins.}

To investigate whether matching AvrPik/Pik combinations were manifested by interactions between their products, a yeast two-hybrid assay was performed. The signal peptide truncated products of seven AvrPik alleles selected were directly interacted with both the full and nucleotide-binding site leucinerich repeat truncated products of all four Pik alleles (Fig. 4A). A pull-down assay confirmed this lack of specificity (Fig. 4B, partial data). Thus, race specificity and recognition specificity appear to be unlinked (Table 3; Fig. 4).

\section{DISCUSSION}

\section{Host selection has shaped}

the genetic population structures of AvrPik alleles.

Compared with neutral genes such as MoDUO1, which was the only polymorphic nucleotide detected in isolate CHL2394 in the GD population, pathogen $A v r$ genes typically have evolved in response to positive selection (Daugherty and Malik 2012; Dodds et al. 2004; Schürch et al. 2004); however, nucleotide diversity tends to be unevenly distributed across the gene sequence. In the case of the barley pathogen Rhynchosporium secalis Avr gene NIP1, the sequence encoding the functional region of the mature protein was approximately four times more variable than the remainder of the sequence, particularly in the signal peptide (Schürch et al. 2004). Similarly, sequence variation in the flax rust Avr gene AvrL567 was concentrated within a 450-bp stretch (Dodds et al. 2004). The potato late blight $A v r$ gene Avrblb1 also possesses a highly diverse region (Champouret et al. 2009), whereas the rice blast Avr gene

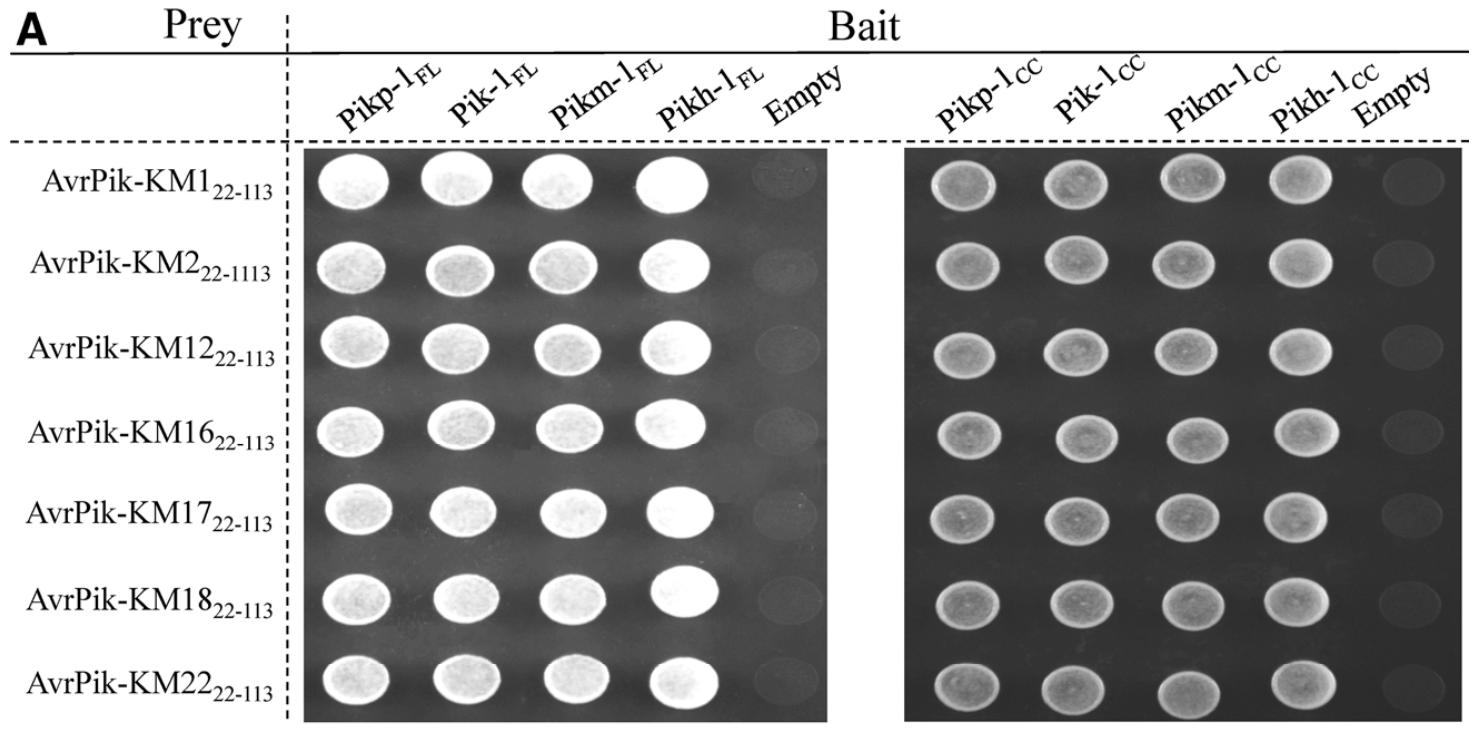

B

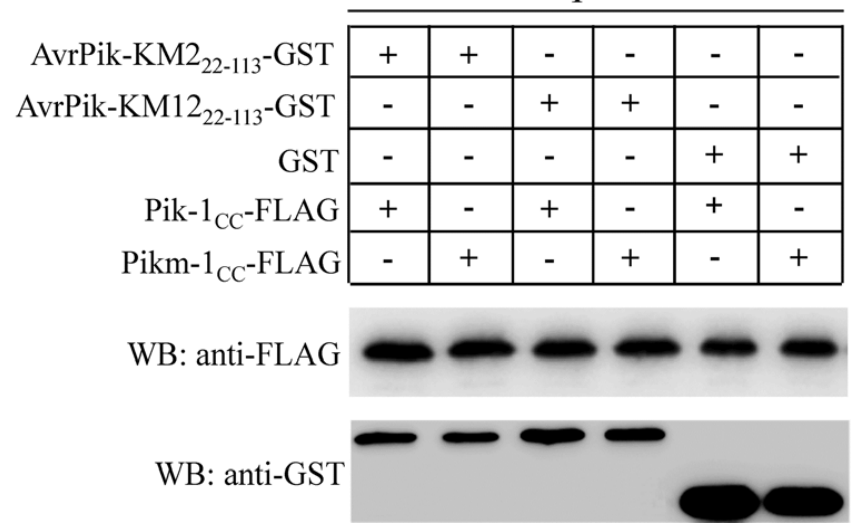

Pull-down

\begin{tabular}{|c|c|c|c|c|c|}
\hline+ & + & - & - & - & - \\
\hline- & - & + & + & - & - \\
\hline- & - & - & - & + & + \\
\hline+ & - & + & - & + & - \\
\hline- & + & - & + & - & + \\
\hline
\end{tabular}
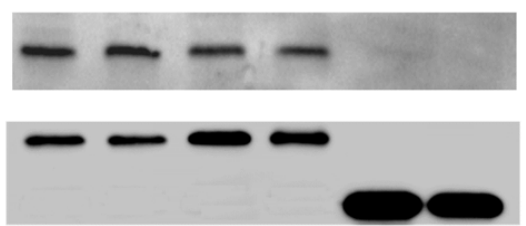

Fig. 4. Interactions between AvrPik and Pik allelic proteins. A, Direct interactions between seven AvrPik and four Pik allelic products, as detected by yeast two-hybrid analysis. AvrPik-KM1, AvrPik-KM2, AvrPik-KM12, AvrPik-KM16, AvrPik-KM17, AvrPik-KM18, and AvrPik-KM22 were selected for the binding specificity test. B, Interactions between two pairs of $A v r P i k$ and $P i k$ allelic proteins, as confirmed by a pull-down system. Western blots employed antiFLAG (Pik-1 ${ }_{\mathrm{CC}}$ and Pikm-1 ${ }_{\mathrm{CC}}$ ) and anti-GST (AvrPik-KM222-113 and AvrPik-KM12 $22-113$ ) antibodies. 
AvrPital proved to be most variable in a specific exon region (Dai et al. 2010). As for the AvrPik alleles described here, positive selection was particularly exerted to the non-signal peptide (non-SP) region rather than to the SP region, indicating that the former (also called the mature protein for AvrPik) is a focus of receiving selection pressure from the cognate $R$ genes. This is consistent with our observation that the mature protein of AvrPik directly interacts with the Pik alleles but the SP containing one does not (Zhai 2012). Furthermore, Yoshida and his colleagues reported that there are both presence-orabsence and nucleotide polymorphisms among AvrPik alleles, of the latter one all are nonsynonymous substitutions, which is a strong evidence of positive selection (Kanzaki et al. 2012; Yoshida et al. 2009). In the current study, we found both nonsynonymous and synonymous substitutions among AvrPik alleles but no presence-or-absence polymorphism in all 240 isolates collected from the four $M$. oryzae populations in China. Thus, $M$. oryzae has adapted to the host selection mainly by rapid amino acid substitution of the AvrPik alleles in such Chinese populations.

The geographical selection for particular AvrPik alleles probably reflects regional differences in the mix of rice cultivars grown. The growing environment in northeastern China is rather similar to that found in Japan, where many cultivars have been bred to carry particular Pik alleles (Xing et al. 1991). As a result, the pathogen population in LN and HLJ will have experienced long-term selection for matching AvrPik alleles in a way that probably would not have occurred in southern China. Rice cultivation in the northeastern region (unlike in the south) has tended to rely on a small number of cultivars: in recent years, the top five most widely grown rice cultivars accounted for $49.9 \%$ of the production area in $\mathrm{LN}$ and $60.4 \%$ in HLJ, while the equivalent proportions in GD and HN were only 20.6 and $35.2 \%$ (Supplementary Table S4). In addition, the rice ecosystem in the northeastern region (one cropping season) is simpler than that in the south (mostly two cropping seasons). Considering the significantly higher genetic diversities of and stronger selection pressure on the AvrPik alleles detected in the northeastern region compared with the southern region (Tables 1 and 2), it appears that host-positive selection rather than geographical and ecological conditions has been the key factor shaping the distribution of AvrPik alleles.

\section{Functional lineages correspond exactly to the pathotypes of AvrPik alleles.}

The relationship between a pathogen's genotype and its pathotype has practical significance for the management of disease (Champouret et al. 2009; Hamer 1991; Zeigler et al. 1995). Although DNA fingerprinting represents an effective means of discriminating between pathogen races, it has not as yet been very successful in predicting pathotype. Although a good relationship based on the analysis of dispersed DNA repeats was claimed to hold among 18 isolates of rice blast (Levy et al. 1991), it was not maintained when an attempt was made to extend it to a larger set of isolates (Xia et al. 1993; Zeigler et al. 1995). Avr gene-specific markers should be better targets for diagnosis because they target the very sequences that specify virulence. A complication, however, is that Avr genes vary not only with respect to their sequence but also with respect to their copy number. For instance, the flax rust gene AvrL567 is present as two copies in avirulent isolates but as just a single copy in virulent ones (Dodds et al. 2004), while the Phytophthora sojae Avrla locus comprises a tandem array of four near-identical copies, two of which are absent in some isolates (Qutob et al. 2009). The well-known AvrPita locus is a family of $M$. grisea species complex, in which AvrPita-1 and AvrPita-2 were present in isolates of both M. oryzae (host plants such as Oryza, Triticum, Eleusine, and Leersia spp.) and $M$. grisea (host plants such as Digitaeria horizontalis and $D$. sangunalis), and AvrPita-3 only in isolates of M. oryzae. The former two function as an $A v r$ gene corresponding to rice $R$ gene Pita, and the latter one does not (Chuma et al. 2011; Khang et al. 2008). As to AvrPik, higher frequencies of isolates sequenced that have two copies in the southern region indicate that copy number variation plays a more important role in the co-evolution process in the southern region compared with that in the northeastern region. Hence, a perfect relationship between genotype and pathotype was only possible when a subset of the 60 functional alleles was used. We can argue that, for an informative relationship to be established between genotype and pathotype, two conditions need to be satisfied: first, the genotype must be based on sequence variation within functional Avr alleles (including their 5' and $3^{\prime}$ flanking regions, if any); and, second, pathotype must be assigned using sets of host lines known to carry single $R$ genes, with a preference for near-isogenic lines which each carry a known $R$ gene. This is a unique report on the demonstration of the relationships between the functional lineages (genotypes) and the Avr-specific pathotypes.

\section{The co-evolution of AvrPik and Pik alleles has been a stepwise process.}

The reconstruction of alleles of the target gene has provided an informative means of assessing functional and evolutionary mechanisms. Lozovsky and associates (2009) reconstructed all 16 possible mutants of the malaria parasite Plasmodium falciparum by combinations of four key amino acids implicated in pyrimethamine resistance. Among 24 evolutionary pathways, three pathways accounting for nearly $90 \%$ of the simulated realizations of the evolution of pyrimethamine resistance were identified. Here, all 16 possible AvrPik alleles referring to the four major amino acids were reconstructed (Fig. 3A), which we believe to be the first application of this technique in a plant pathosystem. Several important insights into the co-evolutionary arms race between AvrPik and Pik alleles were obtained from the network constructed by the global AvrPik alleles.

First, a process of stepwise mutation can explain the evolution of the four alleles detected in the natural pathogen populations: starting from HPGM, the first step consisted of a change to NPGM, then NADM, and finally NADI, thereby matching Pik-p, Pik, Pik-m, and Pik-h, respectively (Fig. 3A). That is, HPGM is most likely the ancestral genotype of AvrPik alleles (Kanzaki et al. 2012). An important question is why they were likely created by only one evolutionary pathway with three mutational steps (Fig. 3A). A rational explanation may be that the cognate Pik alleles, which convey overlapped stepwise resistance spectra (Fig. 1B), sequentially emerged before rice domestication (Pik-s and Pik-p), after rice domestication (Pik and $P i k-m)$, and with modern rice cultivation (Pik-h and Pil) (Fig. 5) (Hua et al. 2012; Zhai 2012; Zhai et al. 2011). This may have led to simple and definitive directional selection pressure on the pathogen AvrPik locus that resulted in generation of a series of AvrPik alleles corresponding to the new Pik alleles as they emerged. This is the simplest and also the most effective evolutionary pathway for creating AvrPik alleles. Because both AvrPik alleles NAGM (1100) on phase II and NADM (1110) on phase III condition the same pathotype (VVAA), the latter one closing to the eventual allele NADI (1111) in the network might have a selective advantage, thereby remaining in the pathogen populations (Fig. 3A). This is a partial reason why there are only three recognizable mutation steps corresponding to the four evolutionary phases in the network for creating the four natural AvrPik alleles. Considering that the 
evolution of a series of AvrPik alleles has mainly been driven by host selection, and the evolution of the host Pik alleles has been a stepwise process (Hua et al. 2012; Zhai 2012; Zhai et al. 2011), the co-evolutionary stepwise relationships between AvrPik and Pik alleles could be established (Fig. 5), in which the three Avr alleles-AvrPik-KM1, AvrPik-KM2, and AvrPikKM12-were perfectly corresponding (virulent) to Pik-s, Pik-p, and Pik, respectively. This, in turn, indicates that both AvrPikKM1 and AvrPik-KM2 emerged before rice domestication, AvrPik-KM12 after rice domestication, and AvrPik-KM16 (virulent to both Pik-m and Pik-h but avirulent to Pil) during modern rice cultivation. Based on our results, the further AvrPik allele, $A v r P i k$ - $\alpha$ (virulent to all six Pik alleles, including Pil) is predicted to emerge in advanced modern rice cultivation. Because there was no allele controlling the Pik-h-specific pathotype, VVVA, which was also not detected in the natural populations tested, AvrPik-KM16 was responsible for both $P i k-m$ and $P i k-h$. Here, we also can argue that, for the stepwise arms race co-evolution relationship to be established between $A v r$ and $R$ genes, three conditions need to be satisfied: first, the pathosystem should comply with the gene-for-gene interaction; second, both $A v r$ and $R$ genes should have series of alleles; and, third, such alleles should convey the stepwise (overlapping) spectrum of phenotypes. Notably, Kanzaki and associates (2012) have recently suggested the co-evolution between AvrPik and $P i k$ that occurred after domestication of rice. It may be due to lacking the defined data of the evolution of Pik alleles in their investigation. Moreover, they have also suggested that the co-evolutionary relationships between AvrPik and Pik alleles were driven by their physical interactions (Kanzaki et al. 2012) but this did not appear to be the case here (Fig. 4; Supplementary Fig. S3). Thus, the linkage between race specificity and recognition specificity in various gene-for-gene pathosystems remains a matter of much debate.

Second, five of the 16 potential AvrPik alleles determined the two non-stepwise pathotypes (VVAV and AVAA), which were not detected in the natural pathogen populations (Fig. 3A). Because both non-stepwise pathotypes were controlled by two blocks of the related alleles in the network, it is indicative that the natural evolution process would be artificially changed by combination of specific SNP. Similarly, various AvrP123 alleles can be differentially recognized by the host genes $P, P 1, P 2$, and $P 3$, and a recombinant allele produced a novel recognition phenotype (Dodds and Thrall 2009; Ravensdale et al. 2012). These, in turn, guide a pathway for engineering the cognate $R$ genes with novel and expanded resistance spectra by combination of the functional SNP (Farnham and Baulcombe 2006; Ravensdale et al. 2012). The further implications of non-stepwise pathotypes for the co-evolution of pathogen and host require a long-term and large-scale investigation of the dynamics of pathogen populations.

Third, race specificities corresponding to the four Pik alleles were presumed by assembling single SNP pairs in the network (Fig. 3B). Only one SNP proved to be the determinant of Pik-p specificity, and single minimal combinations $46+47+48$ and $46+78$ were the determinants for Pik-m and Pik-h, respectively. The situation for $P i k$ was less clear, involving five different combinations. The occurrence of the identical determinant for Pik and Pik-h may reflect the lack of an AvrPik allele matching the $P i k$ - $h$ resistance. Alternatively, there may be an additional SNP implicated in the determination of $P i k-h$ race specificity. This was supported by our previous data that additional AvrPik alleles derived from combinations of the thirdparty mutagenesis (e.g., YPGM and HTGM) of the four major amino acids were also not related to the pathotype (Wu 2012). Similarly, the new AvrPik allele specificity against the youngest and strongest Pik allele, Pil (Hua et al. 2012), would emerge by a combination of new positions on the next evolutionary stage, phase $\mathrm{V}$ (Fig. 5). The multiple combinations were associated with the race specificity for Pik (Fig. 3B), which suggests that this $R$ allele might be placed at the central position for the interaction with and evolution of a series of Pik alleles (Zhai 2012). Further attempts should be focused on reconstruction of the Pik alleles by SNP-directed mutagenesis as well as domain-swapping approaches (Ravensdale et al. 2012; Zhai 2012; Zhai et al. 2011). This, in turn, is believed to improve our understanding of the molecular mechanisms underpinning both race and resistance specificities. This study is, to our knowledge, the first one analyzing the determinants of the $A v r$ allele specificity based on the network reconstructed by all the possible alleles.

A stepwise mutation process has also been demonstrated to be an important mechanism for generating variation in virulence characteristics of other plant pathogens (Hovmøller and

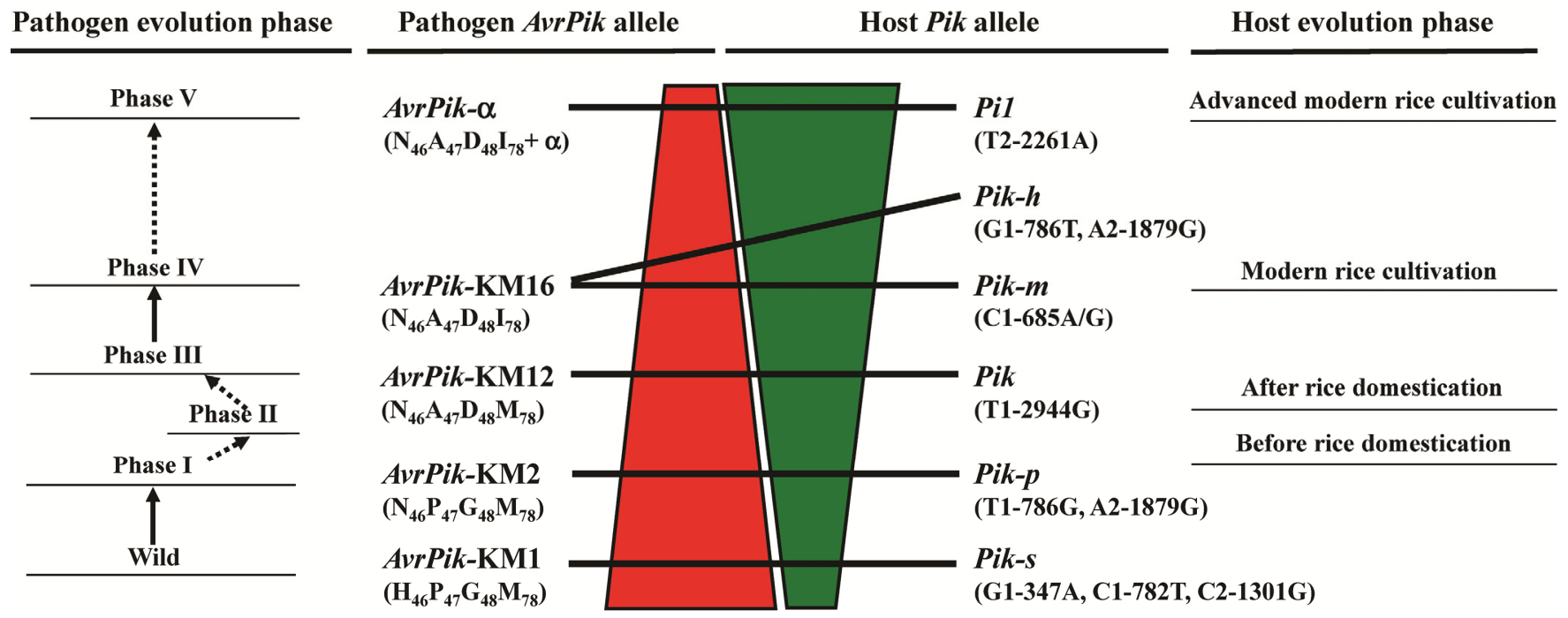

Fig. 5. Co-evolution of AvrPik and Pik reflects a stepwise relationship between alleles. The avirulence spectra of AvrPik alleles and the resistance spectra of $P i k$ alleles were complementarily echeloned by their stepwise mutations, shown in red and green, respectively. The pathogen evolutionary phases, including phase V for creating a further AvrPik allele, AvrPik- $\alpha$, were also indicated. Host evolutionary phases for generating the Pik alleles, including Pi1 and their functional single-nucleotide polymorphisms, were taken from our previous studies (Hua et al. 2012; Zhai 2012; Zhai et al. 2011). 
Justetson 2007; Jiménez-Gasco et al. 2004; Steele et al. 2001; Wang et al. 2007). Without global mutant reconstruction, it was impossible to establish such stepwise relationships in these pathosystems. An intriguing puzzle is the presence of both major and minor mutations at the same locus. A plausible explanation may be that major mutations are unlikely to withstand selection imposed by host $R$ genes, thereby requiring additional minor mutations to create distinct race specificities, accumulatively, for confronting the new $R$ allele-mediated resistance. In fact, such minor mutants mostly occurred in the northeastern region, in which the stronger positive selection pressure existed (Table 3). The problem remains as to how minor mutations can subsequently be converted into major ones. In the case of AvrL567, resistance specificities are mediated by multiple amino acid contacts in a quantitative manner. The evolution of virulent forms of AvrL567 could have occurred in a stepwise manner if a single amino acid change in an avirulent allele resulted in the creation of partially virulent forms endowed with a selective advantage. Subsequent amino acid changes could, then, eventually determine complete virulence (Ravensdale et al. 2012; Wang et al. 2007). In light of this view, we propose that the new stepwise mutation position can emerge in a long-term co-evolutionary arms race process when it becomes complementary and dominant or epistatic to the older ones.

The current data have confirmed and extended our understanding that stepwise mutation is central to the co-evolution between a pathogen and its host. The experimental validation of this and related hypotheses is required for the development of rational and sustainable management strategies against plant disease.

\section{MATERIALS AND METHODS}

\section{Isolate collection and phenotyping.}

The 240 isolates analyzed comprised 60 isolates each from GD, HN (southern China), LN, and HLJ (northeastern China). All isolates were recovered from panicles showing symptoms of rice blast. The isolates were subjected to pathotyping based on four Pik monogenic lines-namely, IRBLkp-K60 (Pik-p), IRBLk-Ka (Pik), IRBLkm-TS (Pik-m), and IRBLkh-K3 (Pik$h$ ) - according to the procedure described by Pan and associates (1996). Each inoculation experiment was conducted at least three times. Because there was no isolate that is avirulent on the host monogenic line IRBLks-F5 carrying Pik-s, it was ruled out in the current study.

\section{SNP marker development and isolate genotyping.}

A generic AvrPik amplicon was obtained with the use of the primer pair $A v r P i k 1 \mathrm{~F} / A v r P i k 1 \mathrm{R}$, designed from the sequence flanking the AvrPik locus. Four further primer pairs $(136 \mathrm{~F} / \mathrm{R}$, $139 \mathrm{~F} / \mathrm{R}, 143 \mathrm{~F} / \mathrm{R}$, and $234 \mathrm{~F} / \mathrm{R}$ ) were designed based on the reference sequence of rice blast isolate Ina168 (Yoshida et al. 2009) and used for dCAPS-based SNP genotyping at positions $136,139,143$, and 234, which were ubiquitous in Chinese isolates tested in the study. Another functional SNP at position 200 (Yoshida et al. 2009), which is a specific one, was ruled out for genotyping and mutant reconstruction. That is, each isolate was subjected to four rounds of SNP assay, and scored as an integrative genotype by a four-digit code. The numerical code indicates the following: 1, PCR product that cannot be digested by the respective enzyme, 2 , PCR product that can be partially digested, and 3, PCR product that can be completely digested. When the genotype was not related to phenotype (pathotype) in a given isolate, it was subjected to TE survey in its $5^{\prime}$ region using the primer pair AvrPik5F/143R. The amplicons and their digested products (if any) were separated by
$8.0 \%$ denaturing polyacrylamide gel electrophoresis and visualized by silver staining.

\section{DNA resequencing and evolution analysis.}

Fifteen representative isolates per geographical population were selected for AvrPik resequencing. Single-species amplicons were subjected to direct sequencing, while multiple-species ones (isolates with double-band-SNP genotypes 2113, 2123, 2223, and 2232) were first cloned individually into pMD20-T (TaKaRa, Dalian, China), and then sequenced. The phylogeny of 60 isolates sequenced was constructed using the Kimura two-parameter model and a maximum likelihood algorithm as facilitated by MEGA v5.0 software. Nucleotide alignments were analyzed by DnaSP v5.0 software. The nucleotide diversity within each domain and population was estimated from both the $\pi$ parameter with the Jukes and Cantor correction (Lynch and Crease 1990), and the $\mathrm{Fu}$ and $\mathrm{Li}(\mathrm{Fu}$ and $\mathrm{Li} 1993) D^{*}$ and $F^{*}$ parameters. The average rates of $\mathrm{Ka}$ and $\mathrm{Ks}$ substitutions were calculated as described by Nei and Gojobori (1986). The coding region of the neutral gene MoDUOI (MGG_02484.6), which is involved in mitosis-related chromosome segregation and is required for the full virulence of the rice blast fungus $M$. oryzae (Peng et al. 2011), was also resequenced for the 60 representative isolates. The sequences of MoDUO1 were used as the controls in the evolution analysis.

\section{Mutant reconstruction.}

Site-directed mutagenesis was performed using a TaKaRa MutanBEST kit. Briefly, AvrPik primers incorporating nonwild-type nucleotides were designed according to the manufacturer's instructions, and PCR products were amplified with Phusion High-Fidelity DNA polymerase (NEB, Beijing), purified, and cloned into pMD20-T for sequencing. Clones containing the desired sequence were double digested with KpnI and PstI and ligated into the binary vector pBHt2, thereby yielding a mutated AvrPik construct. All the mutants detected in the four populations were reconstructed, which includes 16 possible AvrPik alleles with combinations of the four major SNP, and six AvrPik alleles with additional mutations other than the four major SNP. Each construct was then transformed into the field blast isolate CHL724 with virulence on all four Pik alleles, as described by Tosa and associates (2005). Fourteen independent hygromycin-resistant transformants per construct were subjected to pathotyping test.

\section{Yeast two-hybrid assay.}

Because the $P i k$ alleles required the coupled $R$ genes for their functionalities, and the products of Pik-1 alleles but not Pik-2 alleles interact with the cognate pathogen AvrPik proteins truncated with their signal peptides (Kanzaki et al. 2012; Zhai 2012), the full-length and coiled coil (CC) fragments of cDNA corresponding to Pikp-1, Pik-1,Pikm-1, Pikh-1, and the signal peptide-truncated cDNA fragments corresponding to the various AvrPik alleles-(designations in parentheses are from Kanzaki and associates [2012]) AvrPik-KM1 (AvrPik-D), AvrPik-KM2 (AvrPik-E), AvrPik-KM12 (AvrPik-A), AvrPik-KM16 (AvrPikB), AvrPik-KM17 (AvrPik-D-D90N), AvrPik-KM18 (AvrPik-E$A 67 D)$, and AvrPik-KM22 (AvrPik-D-T69A)—were cloned in frame with either the GAL4 DNA binding domain (BD) of the bait vector pGBKT7 or the GAL4 activation domain (AD) of the prey vector $\mathrm{pGADT7}$. The joint transformation of a BD and an $\mathrm{AD}$ construct into $\mathrm{Y} 2 \mathrm{HGold}$ yeast cells was performed using the Matchmaker Gold yeast two-hybrid system (Clontech, Dalian, China), following the manufacturer's instructions. Standard positive (pGBKT7-53 and pGADT7-T) and negative (pGBKT7Lam and pGADT7-T as well as non-allelic blast R protein, Pib) controls were included in each experiment. 


\section{GST pull-down assay.}

Two sets of both AvrPik and Pik allelic proteins were selected. Either the synthetic AvrPik sequence KM2 or KM12 was inserted into the pGEX-6P-1 vector (GE Healthcare, Little Chalfont, U.K.) to form a GST-fusion construct, while the CC fragments of both Pik-1 and Pikm-l fused to a FLAG tag were inserted into a pF3A WG vector (Promega Corp., Madison, WI, U.S.A.). GST-fusion proteins expressed in Escherichia coli strain BL21 were used to pull down the FLAG-fused proteins synthesized in a wheat germ derived in vitro transcription and translation system (Promega Corp.) using a MagneGST pull-down system (Promega Corp.), following the manufacturer's protocol. Expression was detected by Western blotting using anti-FLAG and anti-GST antibodies.

\section{ACKNOWLEDGMENTS}

We thank Z. Zhao (Hunan Rice Research Institute), Z. Liu (Shenyang Agricultural University), and Y. Zhang (Heilongjiang Bayi Agricultural University) for providing the rice blast samples; and T. Mitchell (Ohio State University, U.S.A.) for critical reading of the manuscript. This research was funded by the National Natural Science Foundation (U1131003; 30870102), the National 973 Project (2011CB1007001), and the National Transgenic Plant Project (2011ZX08001-002).

\section{LITERATURE CITED}

Champouret, N., Bouwmeester, K., Rietman, H., van der Lee, T., Maliepaard, C., Heupink, A., van de Voudervoort, P., Jacobsen, E., Visser, R., van der Vossen, E., Govers, F., and Vleeshouwers V. 2009. Phytophthora infestans isolates lacking class I ipiO variants are virulent on Rpi-blb1 potato. Mol. Plant-Microbe Interact. 22:1535-1545.

Chuma, I., Isobe, C., Hotta, Y., Ibaragi, K., Futamata, N., Kusaba, M., Yoshida, K., Terauchi, R., Fujita, Y., Nakayashiki, H., and Valent, B. 2011. Multiple translocation of the AVR-Pita effector gene among chromosomes of the rice blast fungus Magnaporthe oryzae and related species. PLoS Pathog. 7:e1002147. Published online.

Dai, Y., Jia, Y., Correll, J., Wang, X., and Wang, Y. 2010. Diversification and evolution of the avirulence gene AVR-Pital in field isolates of Magnaporthe oryzae. Fungal Genet. Biol. 47:973-980.

Daugherty, M., and Malik, H. 2012. Rules of engagement: Molecular insights from host-virus arms races. Annu. Rev. Genet. 46:677-700.

Dodds, P., and Thrall, P. 2009. Recognition events and host-pathogen coevolution in gene-for-gene resistance to flax rust. Funct. Plant Biol. 36:395-408.

Dodds, P., Lawrence, G., Catanzariti, A., Ayliffe, M., and Ellis, J. 2004. The Melampsora lini AvrL567 avirulence genes are expressed in haustoria and their products are recognized inside plant cells. Plant Cell 16:755-768

Farnham, G., and Baulcombe, D. 2006. Artificial evolution extends the spectrum of viruses that are targeted by a disease-resistance gene from potato. Proc. Natl. Acad. Sci. U.S.A. 103:18828-18833.

$\mathrm{Fu}$, Y., and Li, W. 1993. Statistical tests of neutrality of mutations. Genetics 133:693-709.

Hamer, J. 1991. Molecular probes for rice blast disease. Science 252:632633.

Hovmøller, M., and Justetson, A. 2007. Rates of evolution of avirulence phenotypes and DNA markers in a northwest European population of Puccinia striiformis f. sp. tritici. Mol. Ecol. 16:4637-4647.

Hua, L., Wu, J., Chen, C., Wu, W., He, X., Lin, F., Wang, L., Ashikawa, I., Matsumoto, T., Wang, L., and Pan, Q. 2012. The isolation of Pi1, an allele at the Pik locus which confers broad spectrum resistance to rice blast. Theor. Appl. Genet. 125:1047-1055.

Jiménez-Gasco, M., Milgroom, M., and Jiménez-Díaz, R. 2004. Stepwise evolution of races in Fusarium oxysporum f. sp. ciceris inferred from fingerprinting with repetitive DNA sequences. Phytopathology 94:228235 .

Kanzaki, H., Yoshida, K., Saitoh, H., Fujisaki, K., Hirabuchi, A., Alaux, L., Fournier, E., Tharreau, D., and Terauchi, R. 2012. Arms race co-evolution of Magnaporthe oryzae AvrPik and rice Pik genes driven by their physical interactions. Plant J. 76:894-907.

Khang, C., Park, S., Lee, Y., Valent, B., and Kang, S. 2008. Genome organization and evolution of the AVR-Pita avirulence gene family in the Magnaporthe grisea species complex. Mol. Plant-Microbe Interact. 21:658-670.

Kirzinger, M., and Stavrinides, J. 2012. Host specificity determinants as a genetic continuum. Trends Microbiol. 20:88-93.

Kiyosawa, S. 1974. Studies on genetics and breeding of blast resistance in rice. Misc. Publ. Natl. Inst. Agric. Sci. Ser. D No. 1 (March 1974) 1-58. (In Japanese with English summary)

Kiyosawa, S. 1982. Genetics and epidemiological modeling of breakdown of plant disease resistance. Annu. Rev. Phytopathol. 20:93-117.

Levy, M., Ramao, J., Marchetti, M., and Hamer, J. 1991. DNA fingerprinting with a dispersed repeated sequence resolves pathotype diversity in rice blast fungus. Plant Cell 3:95-112.

Lozovsky, E., Chookajorn, T., Brown, K., Imwong, M., Shaw, P., Kamchonwongpaisan, S., Neafsey, D., Weinreich, D., and Hartl, D. 2009. Stepwise acquisition of pyrimethamine resistance in the malaria parasite. Proc. Natl. Acad. Sci. U.S.A. 106:12025-12030.

Lynch, M., and Crease, T. 1990. The analysis of population survey data on DNA sequence variation. Mol. Biol. Evol. 7:377-394.

Mahuku, G., Jara, C., Cuasquer, J., and Castellanos, G. 2002. Genetic variability within Phaeoisariopsis griseola from central America and its implications for resistance breeding of common bean. Plant Pathol. 51:594-604.

McDonald, B. 1997. The population genetics of fungi: Tools and techniques. Phytopathology 87:448-453.

Nei, M., and Gojobori, T. 1986. Simple methods for estimating the numbers of synonymous and nonsynonymous nucleotide substitutions. Mol. Biol. Evol. 3:418-426.

Pan, Q., Wang, L., Ikehashi, H., and Tanisaka, T. 1996. Identification of a new blast resistance gene in the indica rice cultivar Kasalath using Japanese differential cultivars and isozyme markers. Phytopathology 86:1071-1075.

Peng H., Feng, Y., Zhu, X., Lan, X., Tang, M., Wang, J., Dong, H., and Chen, B. 2011. MoDUO1, a Duo1-like gene, is required for full virulence of the rice blast fungus Magnaporthe oryzae. Curr. Genet. 57:409-420.

Qutob, D., Tedman-Jones, J., Dong, S., Kuflu, K., Pham, H., Wang, Y., Dou, D., Kale, S., Arredondo, F., Tyler, B., and Gijzen, M. 2009. Copy number variation and transcriptional polymorphisms of Phytophthora sojae RXLR effector genes Avrla and Avr3a. PLoS One 4:e5066. Published online.

Raffaele, S., Farrer, R., Cano, L., Studholme, D., Maclean, D., Thines, M., Jiang, R., Zody, M., Kunjeti, S., Donofrio, N., Meyers, B., Nusbaum, C., and Kamoun, S. 2010. Genome evolution following host jumps in the Irish potato famine pathogen lineage. Science 330:1540-1543.

Ravensdale, M., Bernoux, M., Ve, T., Kobe, B., Thrall, P., Ellis, J., and Dodds, P. 2012. Intramolecular interaction influences binding of the flax L5 and L6 resistance proteins to their AvrL567 ligands. PLoS Pathog. 8:e1003004. Published online.

Schürch, S., Linde, C., Knogge, W., Jackson, L., and McDonald, B. 2004. Molecular population genetic analysis differentiates two virulence mechanisms of the fungal avirulence gene NIP1. Mol. Plant-Microbe Interact. 17:1114-1125.

Sirawaraporn, W., Sathitkul, T., Sirawaraporn, R., Yuthavong, Y., and Santi, V. 1997. Antifolate-resistant mutants of Plasmodium falciparum dihydrofolate reductase. Proc. Natl. Acad. Sci. U.S.A. 94:1124-1129.

Steele, K., Humphreys, E., Wellings, C., and Dickinson, M. 2001. Support for a stepwise mutation model for pathogen evolution in Australasian Puccinia striiformis f. sp. tritici by use of molecular markers. Plant Pathol. 50:174-180.

Stukenbrock, E., and McDonald, B. 2009. Population genetics of fungal and Oomycete effectors involved in gene-for-gene interactions. Mol. Plant-Microbe Interact. 22:371-380.

Terauchi, R, and Yoshida, K. 2010. Towards population genomics of effector-effector target interactions. New Phytol. 187:929-939.

Tosa, Y., Osue, J., Eto, Y., Oh, H., Nakayashiki, H., Mayama, S., and Leong, S. 2005. Evolution of an avirulence gene, AVR1-CO39, concomitant with the evolution and differentiation of Magnaporthe oryzae. Mol. Plant-Microbe Interact. 18:1148-1160.

Wang, C., Guncar, G., Forwood, J., The, T., Catanzariti, A., Lawrence, G., Loughlin, F., Mackay, J., Schirra, H., Anderson, P., Ellis, J., Doddes, P., and Kobe, B. 2007. Crystal structures of flax rust avirulence proteins AvrL567-A and -D reveal details of the structural basis for flax disease resistance specificity. Plant Cell 19:2898-2912.

Wang, L., Xu, X., Lin, F., and Pan, Q. 2009. Characterization of rice blast resistance genes in the Pik cluster and fine mapping of the Pik-p locus. Phytopathology 99:900-905.

Wu, W. 2012. Genetic diversity and evolution of the AvrPik alleles in the rice blast pathogen populations. Ph.D. thesis, South China Agricultural University, Guangzhou, China.

Xia, J., Correll, J., Lee, F., Marchetti, M., and Rhoads, D. 1993. DNA fingerprinting to examine microgeographic variation in the Magnaporthe grisea (Pyricularia grisea) population in two rice fields in Arkansas. Phytopathology 83:1029-1035. 
Xing, Z., Que, G., and Li, C. 1991. Early japonica rice. Pages 106-138 in: Rice Varieties and their Genealogy in China. S. Lin and S. Min, eds. Shanghai Scientific and Technical Publishers, Shanghai, China. (In Chinese).

Yoshida, K., Siatoh, H., Fujisawa, S., Kanzaki, H., Matsumura, H., Yoshida, K., Tosa, Y., Chuma, I., Takano, Y., Win, J., Kamoun, S., and Terauchi, R. 2009. Association genetics reveals three novel avirulence genes from the rice blast fungal pathogen Magnaporthe oryzae. Plant Cell 21:15731591.

Zeigler, R., Cuoc, L., Scott, R., Bernardo, M., Chen, D., Valent, B., and Nelson, R. 1995. The relationship between lineage and virulence in Pyricularia grisea in the Philippines. Phytopathology 85:443-451.

Zhai, C. 2012. Function, evolution and interaction of the Pik gene family conferring resistance to Magnaporthe oryzae. Ph.D. thesis, South China Agricultural University, Guangzhou, China.

Zhai, C., Lin, F., Dong, Z., He, X., Yuan, B., Zeng, X., Wang, L., and Pan Q. 2011. The isolation and characterization of Pik, a rice blast resistance gene which emerged after rice domestication. New Phytol $189: 321-334$

AUTHOR-RECOMMENDED INTERNET RESOURCES

dCAPS program: helix.wustl.edu/dcaps/dcaps.html

MEGA software: www.megasoftware.net

DnaSP sequence polymorphism software: www.ub.edu/dnasp

Figure $3 \mathrm{~B}$ was corrected and replaced on July 31, 2015. 